\title{
Síndrome de Down en otorrinolaringología
}

\author{
Down Syndrome in Otolaryngology
}

\author{
Jorge Zúñiga P1, Magdalena Raggio P².
}

\begin{abstract}
RESUMEN
El síndrome de Down es la cromosomopatía más frecuente en Chile. Los pacientes portadores de este síndrome presentan una serie de dismorfias y malformaciones congénitas. Muchas patologías secundarias a estos trastornos son de la esfera otorrinolaringológica. Existen alteraciones otológicas, rinosinusitis crónica, apnea obstructiva del sueño, obstrucción de la vía aérea, inestabilidad atlantoaxial, etc.

Se realiza una revisión bibliográfica y se describen las patologías más relevantes y su enfrentamiento.

Palabras clave: Síndrome de Down, estenosis subglótica, inestabilidad atlantoaxial.

\section{ABSTRACT}

Down syndrome is the most common chromosomal abnormality in Chile. The patients with this syndrome have a number of dysmorphic features and congenital malformations. Many secondary pathologies of these disorders are ENT sphere. There otologic disorders, chronic rhinosinusitis, obstructive sleep apnea, airway obstruction, atlantoaxial instability, etc.

A literature review is performed and the most relevant diseases and confrontation are described.
\end{abstract}

Key words: Down syndrome, pediatric otolaryngology, otology.

10torrinolaringólogo, Hospital Clínico de la Universidad de Chile - Clínica Santa María.

${ }^{2}$ Interna Medicina, Facultad de Medicina, Universidad de Chile.

Recibido el 19 de septiembre, 2014. Aceptado el 30 de diciembre, 2014. 


\section{INTRODUCCIÓN}

El síndrome de Down (SD) es la cromosomopatía más frecuente en recién nacidos en Chile. La tasa promedio para el período 1998-2005 fue de 2,93 casos por 1.000 nacidos vivos, siendo esta tasa muy alta cuando se compara con el ECLAMC (Estudio Colaborativo Latinoamericano de Malformaciones Congénitas), cuya tasa es 1,88 por 1.000 nacidos vivos.

Son características del síndrome de Down una serie de dismorfias y malformaciones congénitas, que los distinguen fenotípicamente y además predisponen a los niños con síndrome de Down a un gran número de patologías agregadas ${ }^{1}$.

Un significativo número de estas patologías agregadas tienen lugar en el área otorrinolaringológica, por lo cual, es de gran importancia para la especialidad conocer las características del síndrome de Down y también conocer su problemática más frecuente. Este conocimiento permitirá prevenir y tener un impacto significativo en el desarrollo que el niño pueda alcanzar.

\section{ALTERACIONES CRANEOFACIALES}

Los niños con SD presentan dorso nasal aplanado, hipoplasia mediofacial y micrognatia.

Pueden además tener orejas pequeñas, plegadas 0 displásicas. También pueden tener malformaciones en la tuba auditiva y en el oído interno, macroglosia, paladar estrecho, faringe estrecha.

A estas malformaciones, se le agrega la hipotonía generalizada, condición que contribuye a la ocurrencia de problemas otorrinolaringológicos ${ }^{2,3}$.

\section{OTOLOGÍA}

En el área de la otología existen una serie de patologías de gran importancia en el niño con SD.

La estenosis del meato auditivo externo (CAE) se presenta entre el $40 \%$ y $50 \%$ de los niños con $\mathrm{SD}$, lo cual predispone a la formación de tapones de cerumen, impactación de cuerpos extraños que son más difíciles de extraer, y causa hipoacusia de conducción. Además un CAE estrecho puede esconder la presencia de colesteatomas, permitiendo así el avance de esta patología sin tratamiento oportuno, significando un daño irreparable en el oído del niño $0^{4,5}$.

La otitis media con efusión (OME) es una patología de prevalencia elevada (sobre el $80 \%$ ) en pacientes $\mathrm{SD}^{6}$. Noventa y tres por ciento presenta un episodio durante el primer año de vida, y el $68 \%$ en el quinto año $0^{7}$.

Su etiología puede estar determinada anatómicamente por la hipoplasia mediofacial con una nasofaringe estrecha y un adenoides relativamente grande, junto a una inserción anormal de la tuba auditiva 0 a un mal funcionamiento de la musculatura que la abre.

Existen estudios además que demuestran una consistente hipoplasia de la lámina lateral cartilaginosa de la tuba en pacientes con SD, lo que traduce un volumen menor en la lámina y un radio de inserción menor del músculo tensor del velo del paladar, además de un menor radio del lumen de la tuba ${ }^{8-10}$. Todos estos factores, asociados a las infecciones a repetición, son causantes de que la OME sea tan frecuente en niños con síndrome de Down.

La inserción de tubos de ventilación puede ser muy difícil, y menos efectivas por la estrechez del meato auditivo externo, la elevada incidencia de otorrea persistente y por el riesgo aumentado de perforación timpánica persistente luego de la salida del tubo, por la ausencia de lámina propia en el tímpano.

Cuatro por ciento de los niños con SD presentan hipoacusia sensorioneural al momento del screening de recién nacidos, ya sea unilateral 0 bilateral ${ }^{13}$. Se ha visto que con la edad se produce un aumento de su prevalencia, llegando hasta el $20 \%$ en adolescentes y adultos jóvenes.

Se ha visto además un desarrollo prematuro de presbiacusia, pudiendo manifestarse hasta 30 ó 40 años antes que el promedio de la población ${ }^{14-15}$.

Existen estudios que demuestran que puede existir hipoplasia del oído interno en pacientes con SD: meato auditivo interno estrecho, estrechez crítica del canal del nervio coclear, cóclea pequeña, modiolo, canal semicircular lateral y vestíbulos más pequeños de lo encontrado en la población general. Además, se ha visto duplicación del meato auditivo interno, malformación de Mondini, saco endolinfático dilatado, acueducto del vestíbulo elongado y canal semicircular lateral con diámetro disminuido ${ }^{17}$. 


\section{RINOSINUSOLOGÍA}

La rinosinusitis crónica es una patología común en los niños con SD, que está determinada por una hiperplasia adenoidea, alergia e infección sinusal. En estos niños, la hipoplasia del tercio medio de la cara produce acumulación y estancamiento del mucus, favoreciendo la sobreinfección ${ }^{18}$.

\section{SAHOS (SÍNDROME DE APNEA E HIPOAPNEA OBSTRUCTIVA DEL SUEÑO)}

Más de un tercio de los pacientes con SD sufren esta patología, cuya aparición se ve favorecida por múltiples factores: La pequeña longitud anteroposterior de nasofaringe y orofaringe, la hiperplasia adenoamigdalina, la hipotonía muscular, el reflujo faringolaríngeo y la obesidad ${ }^{19,20}$. Al ser tan frecuente en estos pacientes, se debe pesquisar activamente el mal dormir, la sudoración nocturna, los ronquidos, la somnolencia diurna, los despertares frecuentes, las pausas respiratorias y la aparición de cianosis. En relación a esta patología, el beneficio de la adenoamigdalectomía en niños con SD es significativo, pero las apneas nocturnas pueden persistir o recurrir. Si esto ocurre, se debe plantear que exista una hiperplasia de la amígdala lingual, siendo beneficiosa su resección. En casos que sean refractarios, será beneficioso el uso de CPAP21,22.

\section{OBSTRUCCIÓN DE VÍA AÉREA}

Los niños con SD presentan alteraciones de la vía aérea que facilitan la obstrucción de ésta, especialmente de la vía aérea superior. La prevalencia de la laringomalacia se eleva hasta el $43 \%$, y son frecuentes también la traqueomalacia y la estenosis traqueal|23,24.

Los pacientes con SD presentan en un grado casi universal estenosis subglótica congénita. Más de la mitad son asintomáticos, pero pueden producirse episodios frecuentes de croup, presentar intubación difícil o descompensarse después de la extubación. Es de muy difícil manejo cuando es una estenosis sintomática o secundaria a intubación prolongada. Es frecuente además encontrar anillos traqueales en estos pacientes ${ }^{25}$.

\section{INESTABILIDAD ATLANTO-AXIAL}

Esta condición afecta el 30\% de los niños con el síndrome ${ }^{26}$. En las radiografías cervicales se aprecia que entre el $20 \%$ y $30 \%$ presenta una distancia anormalmente aumentada entre el arco del atlas y el proceso odontoides del axis (mayor a 4,5 $\mathrm{mm}$ ). A pesar de eso la imagenología tiene pobres resultados para diagnosticar un compromiso clínico neurológico. Para solucionar esto se realizan potenciales evocados somatosensoriales (SEP), éstos miden la rapidez y eficiencia de la transmisión neural aferente y procesamiento. Pueschel y Scola encontraron un importante retraso en las latencias SEP en pacientes con un espacio atlanto-odontoídeo mayor a $5 \mathrm{~mm}$ en posición neutral de la cabeza ${ }^{27}$.

Pacientes con una distancia de $4,5 \mathrm{~mm} 0 \mathrm{me}$ nor no tienen problemas en una rotación de cabeza lateral en $60^{\circ}$, medido por SEP28.

La inestabilidad también puede ser atlantooccipital29.

Especial cuidado debe tenerse con la hiperextensión o rotación del cuello en la cirugía de adenoamigdalectomía y punción timpánica²6.

\section{HIPOTIROIDISMO}

El hipotiroidismo congénito es 28 veces más frecuente en niños con SD que en la población general. Es frecuente su presentación subclínica, por lo cual es importante realizar pruebas de hormonas tiroideas y anticuerpos antitiroideos periódicamente (en el Reino Unido es cada dos años) $)^{30}$.

\section{LENGUAJE Y VOZ}

El desarrollo del lenguaje está alterado en los niños con SD. Esto es multifactorial, pudiendo estar determinado por una baja auditiva, dificultades en el aprendizaje, y déficits particulares en el desarrollo del habla y el lenguaje. Además, se ha visto una alta tasa de alteraciones articulatorias en comparación con otros niños ${ }^{31}$. El control muscular de la lengua, la coordinación entre labios y lengua y la planificación de secuencias motoras están deterioradas en estos pacientes, dando 
lugar a disartria, dispraxia, problemas de disfagia y articulación. Además, la hipotonía muscular de la laringe predispone a fatiga muscular, lo que se traduce en voces roncas y monótonas, tensas, guturales de tono bajo y estridente. Son también descritas como nasales, susurradas y ásperas ${ }^{32,33}$.

Se ha visto un marcado retraso en el balbuceo, en la aparición de solicitudes no verbales, y un deterioro en el lenguaje expresivo, tanto en el vocabulario como en la sintaxis. Estos siguen retardados en la adolescencia, agregándose deterioro de la comprensión y la recepción del idioma ${ }^{34}$.

\section{REFLUJO GASTROESOFÁGICO}

La enfermedad por reflujo gastroesofágico y la acalasia son patologías con mayor frecuencia en la población con SD ${ }^{35}$. Síntomas como pirosis, regurgitación y dolor torácico no cardiaco deben ser buscados, ya que la dificultad en el lenguaje y la comunicación en estos pacientes pueden retrasar significativamente el diagnóstico.

\section{CONCLUSIÓN}

El paciente portador del síndrome de Down tiene una serie de alteraciones en la esfera otorrinolaringológica que resultan importantes en su desarrollo y su calidad de vida.
Debemos pesquisar dirigidamente muchas de las patologías sobre todo las que pueden ser subclínicas.

Pensamos que existen algunos enfrentamientos que no deben olvidarse y los presentamos en la Tabla 1.

\section{BIBLIOGRAFÍA}

1. Nazer J, Cifuentes L. Estudio epidemiológico global del Sd de Down. Rev Chil Pediatr 2011; 82(2): 105-12.

2. Farkas LG, Katic MJ, Forest CR, litsas L. Surface anatomy of the face in Down syndrome: linear and angular measurements in the craniofacial regions. J Craniofac Surg 2001; 129(4): 373-9.

3. Shott SR, Amin R, Chini B, Heubi C, Hotze S, AKers R. Obstructive sleep apnea: should all children with Down syndrome be tested? Arch Otolaryngol 2006; 132: 432-6.

4. Sнотт S. Down syndrome: common otolaryngologic manifestations. Am J Med Genet C 2006; 42C: 131-40.

5. Bacciu A, Pasanisi E, Vincenti V, Giordano D, Caruso A, Lauda L, Bacciu S. Surgical treatment of middle ear cholesteatoma in children with Down syndrome. Otol Neurotol 2005; 26: 1007-10.

6. Shott S, Joseph A \& Heithaus D. Hearing loss in Down syndrome. Int J Paediatr Otorhinolaryngol 2001; 61: 199-205.

Tabla 1. Recomendaciones para la consulta de un paciente portador de un síndrome de Down

\begin{tabular}{|l|l|}
\hline 1. & $\begin{array}{l}\text { Revisar sus oídos, la presencia de estenosis de CAE, pesquisar la presencia de otitis media con efusión, } \\
\text { preguntar a sus padres por hipoacusia. }\end{array}$ \\
\hline 2. & $\begin{array}{l}\text { Controlar precozmente la audición del niño, al nacimiento, luego cada } 6 \text { meses hasta los dos años, para } \\
\text { continuar con evaluación anual. }\end{array}$ \\
\hline 3. & $\begin{array}{l}\text { Preguntar a los padres dirigidamente por problemas para dormir, roncopatía, pausas respiratorias nocturnas, } \\
\text { enuresis, sudoración nocturna, somnolencia diurna. }\end{array}$ \\
\hline 4. & Realizar una evaluación del lenguaje, derivar precozmente al fonoaudiólogo si se estima necesario. \\
\hline 5. & Evaluar función tiroidea si esto no ha sido realizado. \\
\hline 6. & Consultar a sus padres por infecciones nasales a repetición, pedir TC de CPN de ser necesario. \\
\hline 7. & $\begin{array}{l}\text { Si es necesario intervenir quirúrgicamente al niño, evaluar siempre estabilidad de articulación atlanto-axial, } \\
\text { pedir radiografía cervical, y evitar la rotación cervical en cirugías de oído. }\end{array}$ \\
\hline
\end{tabular}


7. Barr E, Dungworth J, Hunter $\mathrm{K}$ et al. The prevalence of ear, nose and throat disorders in preschool children with Down's syndrome in Glasgow. Scott Med J 2011; 56, 98-103.

8. Verstegen RJ, Kusters MAA, Gemen EFA, DE Vries E. Down syndrome B-lymphocyte subpopulations, intrinsic defect or decreased T-lymphocyte help. Pediatr Res 2010; 67(5): 563-9.

9. Sнотт S. Down syndrome: common otolaryngologic manifestations. Am J Med Genet C 2006; 42C: $131-40$.

10. Miura M, Sando I, Balaban C, Haginiomori SI, Orita Y. Temporal bone 3 morphometric study on the eustachian tube and its associated structures in patients with chromosomal aberrations. Ann Otol Rhinol Laryngol 2002; 111(8): 722-9.

11. I Ino Y, Imamura Y, Harigal S et al. Efficacy of tympanostomy tube insertion for otitis media with effusion in children with Down syndrome. Int $J$ Paediatr Otorhinolaryngol 1999; 49: 143-9.

12. Sheehan PZ \& Hans PS. UK and Ireland experience of bone anchored hearing aids (BAHA) in individuals with Down syndrome. Int J Paediatr Otorhinolaryngol 2006; 70: 981-6.

13. Park AH, Wilson MA, Stevens PT et al. Identification of hearing loss in pediatric patients with Down syndrome. Otolaryngol. Head Neck Surg 2012; 146: $135-40$.

14. Dahle AJ \& McCollister FP. Hearing loss and otologic disorders in children with Down syndrome. Am J Ment Defic 1986; 90: 636-42.

15. FuLTon RT \& LLoYd LL. Hearing impairment in a population of children with Down's syndrome. Am J Ment Defic 1968; 73: 298-302.

16. Guidelines for essential medial surveillance. http://www.dsmig.org.uk [accessed on 16 April 2012].

17. Susan Blaser, Evan J Propst, Daniel Martin, Annette Feigenbaum, Adrian L James, Patrick Shannon, Blake C PAPSin. Inner Ear Dysplasia is Common in Children With Down Syndrome (trisomy 21). Laryngoscope 2006; 116: 2113-9.

18. Mitchell RB, Call E \& Kelly J. Ear, nose and throat disorders in children with Down syndrome. Laryngoscope 2003; 113: 259-63.

19. Shott SR, Amin R, Chin B et Al. Obstructive sleep apnea: should all children with Down syndrome be tested? Arch Otolaryngol Head Neck Surg 2006; 132: 432-6.
20. Suskind DL, Zeringue GP, Kluka EA et al. Gastroesophageal reflux and paediatric otolaryngologic disease: the role of antireflux surgery. Arch Otolaryngol Head Neck Surg 2001; 127: 511-4.

21. Barr E, Dungworth J, Hunter K et al. The prevalence of ear, nose and throat disorders in preschool children with Down's syndrome in Glasgow. Scott Med J 2011; 56: 98-103.

22. Abdel-Aziz M, Ibrahim N, Ahmed A et al. Lingual tonsils hypertrophy; a cause of obstructive sleep apnoea in children after adenotonsillectomy: operative problems and management. Int $J$ Pediatr Otorhinolaryngol 2011; 75: 1127-31.

23. Mitchell RB, Call E, Kelly J. Diagnosis and therapy for airway obstruction in children with Down syndrome. Arch Otolaryngol 2003; 129: 642-5.

24. Bertrand P, Navarro $\mathrm{H}$, Caussade $\mathrm{H}$, Holmgren N, SÁnchez I. Airway anomalies in children with Down syndrome: endoscopic findings. Pediatr Pulm 2003; 36: 137-41.

25. Miller R, Gray SD, Cotton RT et al. Subglottic stenosis and Down syndrome. Am J Otolaryngol 1990; 11: 274-7.

26. Hankinson TC \& Anderson RC. Craniovertebral junction abnormalities in Down syndrome. Neurosurgery 2010; 66 (3 Suppl): 32-8.

27. Pueschel SM, Scola FH. Atlantoaxial instability in individuals with Down syndrome: epidemiologic, radiographic, and clinical studies. Pediatrics 1987; 80: 555-60. American Academy of Pediatrics, Committee on Sports Medicine and Fitness: Atlantoaxial instability in Down syndrome: subject review. Pediatrics 1995; 96: 151-4.

28. N Wendell Todd, Philip J Holt, A Thomas Allen. Safety of Neck Rotation for Ear Surgery in Children With Down Syndrome. Laryngoscope 2000; 110: 1442-5.

29. Tredwell SJ, Newman DE, Lockitch G. Instability of the upper cervical spine in Down syndrome. J Pediat rOrthop 1990; 10: 602-6.

30. Fort P, Lifshitz F, Bellisario R et al. Abnormalities of thyroid function in infants with Down syndrome. J Paediatr 1994; 104: 545-9.

31. LELANd J, Wood S, Hardcastle W, Wishart J, Timmins C. Relationship between speech, oromotor, language and cognitive abilities in children with Down's syndrome. Int J Lang Commun Disord 2010; 45: 83-95. 
32. Venail F, Gardiner Q, Mondain M. ENT and speech disorders in children with Down's syndrome: an overview of pathophysiology, clinical features, treatments, and current management. Clin Pediatr 2004; 43(9): 783-91.

33. Moura CP, Cunha LM, Vilarinho H, Cunha MJ, Freitas D, Palha M, Pueschel SM, Pais-Clemente M. Voice parameters in children with Down syndrome. J Voice 2006; 22(1): 34-42.
34. Venail F, Gardiner Q, Mondain M. ENT and speech disorders in children with Down's syndrome: an overview of pathophysiology, clinical features, treatments, and current management. Clin Pediatr 2004; 43(9): 783-91.

35. Zárate N, Mearin F, Hidalgo A, Malagelada JR. Prospective evaluation of esophageal motor dysfunction in Down's syndrome. Am J Gastroenterol 2001; 96(6): 1718-24. 